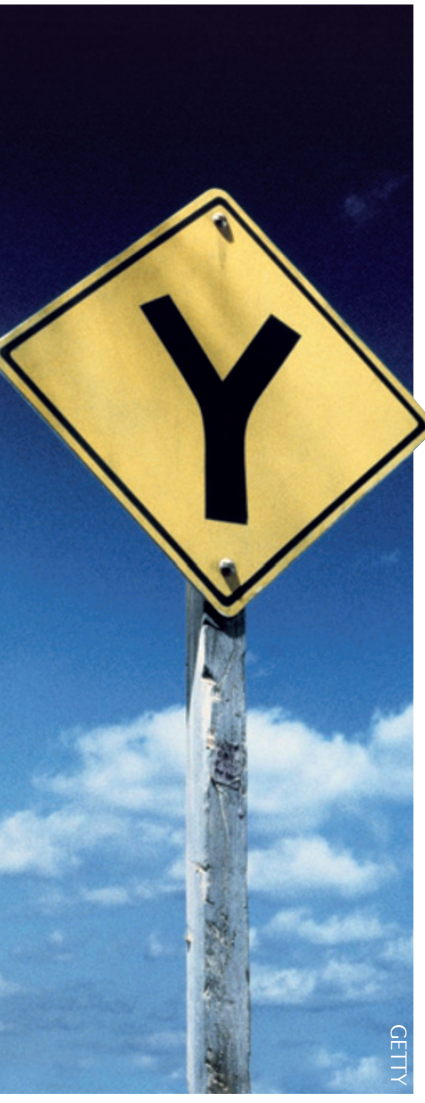

EVOLUTION

\section{Converging on divergence}

Although bacterial populations reproduce asexually by clonal expansion, horizontal gene transfer (HGT) of advantageous genetic elements represents a pathway for divergence that is akin to sexual evolution. However, the impact of HGT on ecological differentiation is controversial: the rarity of homologous recombination is predicted to restrict the separation of a gene from the rest of the genome, but the spread of individual genes and alleles through populations (gene-specific sweeps) is frequently observed. Now, Shapiro et al. reconcile these seemingly conflicting observations by showing that during the early stages of ecological differentiation, individual genes and genome regions can sweep through bacterial populations, but in a manner that eventually favours the stabilization of genetically similar populations within the same habitat.
The Vibrio cyclitrophicus genome consists of two chromosomes, which are each composed of core regions that are shared by all isolates and flexible regions that vary between isolates. To assess the early events in ecological differentiation, the authors sequenced the genomes of isolates from two populations that have only recently diverged and now occupy distinct microhabitats in the ocean. Surprisingly, they found that the core genome was dominated by SNPs that were present in both populations, suggesting that rather than arising solely by clonal descent, there was a high degree of gene flow between the two emerging populations despite their ecological dispersal.

So if HGT between spatially segregated populations is common, how do distinct populations emerge? The authors found that a large region of chromosome II had swept through most of the isolates in one population but was not found in the other, providing evidence of an ecologically partitioned selective sweep. Furthermore, in very recently diverged isolates, or 'sister strains', most recent recombination events resulted from pairing between strains of the same population, suggesting a substantial bias towards higher recombination rates among strains that occupied the same habitat.

The authors suggest that although gene flow between the populations appears to be rife at early time points, the acquisition of habitat-specific genes is likely to instigate ecological separation, and this is gradually enhanced by biased recombination within, rather than between, segregating populations.

Christina Tobin Kåhrström, Associate Editor, Nature Reviews Microbiology

This article is modified from the original in Nature Rev. Microbiol. (doi:10.1038/nrmicro2798)

ORIGINAL RESEARCH PAPER Shapiro, B. J. et al. Population genomics of early events in the ecological differentiation of bacteria. Science 336 , 48-51 (2012) 